\title{
Role of postoperative radiotherapy in reducing ipsilateral recurrence in DCIS: an observational study of 1048 cases
}

Stefanie Corradini ${ }^{*}$ (D), Montserrat Pazos ${ }^{1}$, Stephan Schönecker ${ }^{1}$, Daniel Reitz ${ }^{1}$, Maximilian Niyazi ${ }^{1}$, Ute Ganswindt ${ }^{1,2}$, Simone Schrodi ${ }^{3}$, Michael Braun ${ }^{4}$, Martin Pölcher ${ }^{4}$, Sven Mahner ${ }^{5,6}$, Nadia Harbeck ${ }^{5,6}$, Jutta Engel ${ }^{3}$ and Claus Belka ${ }^{1,6}$

\begin{abstract}
Background: The objective of the present study was to evaluate the effectiveness of postoperative radiotherapy after breast conserving surgery (BCS) in DCIS in a large patient population treated in clinical practice.

Methods: Data were provided by the population-based Munich Cancer Registry. Between 1998 and 2014, 1048 female patients with diagnosis of DCIS and treated at two Breast Care Centres were included in this observational study. The effectiveness of postoperative radiotherapy and variables predicting the use of radiotherapy were retrospectively analysed.
\end{abstract}

Results: After adjusting for age, tumour characteristics and therapies, Cox regression analysis for local recurrence-free survival identified RT as an independent predictor for improved local control (HR: 0.579; 95\%Cl: 0.384-0.872, p = 0.008). Ten-year cumulative incidence of in-breast recurrences was $20.0 \%$ following BCS, compared to $13.6 \%$ in patients receiving postoperative radiotherapy $(p=0.012)$. As an estimate for disease-specific survival, 10-year relative survival was $105.4 \%$ for patients receiving postoperative radiotherapy and 101.6\% without radiotherapy. On multivariate analysis, postoperative radiotherapy was not associated with improved overall survival (HR 0.526; 95\%Cl: 0.263-1.052, $p=0.069$ ). Over time, a significant increase of RT was registered: while 1998 only $42.9 \%$ of patients received postoperative radiotherapy, the proportion rose to $91.2 \%$ in 2014 . Women aged < 50 years (OR: 2.559, 95\%Cl: 1.416-4.625, $p<0$. 001 ) or with negative hormone receptor status (OR: 2.625, 95\% Cl: $1.458-4.728, p=0.001$ ) or receiving endocrine therapy (OR: $1.762,95 \% \mathrm{Cl}: 1.060-2.927, p=0.029)$ were more likely to receive postoperative radiotherapy after BCS.

Conclusions: In conclusion, this study provides insights regarding the adoption and treatment pattern of postoperative RT following BCS for DCIS in a large cohort reflecting "real-life" clinical practice in this setting. Postoperative RT was found to be associated with a reduced risk of ipsilateral recurrence and no survival benefit compared to observation alone.

Keywords: Ductal carcinoma in situ, Breast conserving surgery, Radiotherapy, Local control, In-breast recurrence, Survival, Outcome

\footnotetext{
* Correspondence: Stefanie.corradini@med.uni-muenchen.de

'Department of Radiation Oncology, University Hospital, LMU Munich,

Marchioninistraße 15, 81377 Munich, Germany

Full list of author information is available at the end of the article
} 


\section{Background}

The optimal management of ductal carcinoma in situ (DCIS) of the breast is still controversial and to date, inconsistent and conflicting results have been reported across prospective and observational studies [1]. As known from several randomised trials and a large EBCTCG metaanalysis [2-6] postoperative radiotherapy (RT) after breast conserving surgery is a very effective adjuvant local treatment modality. Radiotherapy approximately halves the risk of local recurrences [6] as compared to surgical resection alone, with similar effects in reducing ipsilateral in situ and invasive recurrences [4]. This effect was observed in all subgroups of patients and could not be translated into a survival benefit in randomised studies [6]. Therefore, over the past years a risk-benefit debate for or against postoperative radiotherapy has attracted wide public attention $[1,7]$.

DCIS of the breast represents a very heterogeneous disease, making a risk-group stratification challenging. Considerable efforts have been spent in the implementation of risk-adapted treatment concepts. One major objective was to identify a subset of "low-risk" patients with excellent prognosis, where a treatment de-escalation with omission of radiotherapy can be safely attempted, as recurrence rates are very low $[8,9]$. On the other hand, considerations about a possible undertreatment of "high-risk" patients have led to treatment escalation strategies using boost irradiation to the tumour bed to further increase local control rates [10, 11].

The above-mentioned uncertainties result in a vast heterogeneity of criteria influencing treatment decisionmaking in real-world settings. Clinicians have to choose from a range of available risk stratification tools [12-14] and treatment options in order to select a suitable treatment strategy for each individual patient. Furthermore, shared decision-making in daily clinical practice is strongly influenced by a number of confounding factors, such as clinician and patient preferences and trade-offs concerning toxicity risks or comorbidities [15-17]. The aim of the present study was to evaluate the effectiveness of postoperative radiotherapy in DCIS in a large patient population, outside of clinical trials. Moreover, the study aimed to identify possible factors related to the use of postoperative radiotherapy in daily clinical practice.

\section{Methods}

\section{Data sources}

Data were retrieved from the Munich Cancer Registry (MCR) [18]. The catchment area of the populationbased clinical cancer registry to date encompasses 4.81 million inhabitants in southern Germany. Over the past decades it has been stepwise enlarged from 2.3 million inhabitants in 1998 to 3.84 million in 2002, and nowadays includes all regions of Upper Bavaria. The MCR systematically retrieves cancer notifications from
73 MCR-affiliated hospitals or other notifying institutions. Patient's demographics, cancer diagnosis, disease and treatment characteristics and follow-up are reported following official documentation guidelines for cancer registries [19, 20]. Follow-up was conducted following the national German "S3-guideline" for breast cancer [21], with regularly clinical and mammographic followups. All follow-up data were provided as cancer notifications from MCR-affiliated hospitals or other notifying institutions on a regular basis and collected prospectively at the MCR. Moreover, all pathology laboratories within the catchment area of the $\mathrm{MCR}$ are required to submit their pathology reports to the cancer registry. Thus, the total number of pathologically diagnosed recurrences within the catchment area was systematically obtained. Survival information is maintained systematically from death certificates of 23 health offices within the catchment area of the MCR.

\section{Study population}

For the present study, patients treated at the Red Cross Breast Centre and the LMU Breast Centre in Munich (Germany) between 1998 and 2014 were analysed. Of all breast malignancy records, male patients $(n=63)$, cases with histology of lymphoma $(n=10)$, sarcoma $(n=58)$ or invasive carcinoma $(n=13,064)$, or with unknown date of initial diagnosis (e.g. tumors from death certificate information only [DCO]) $(n=58)$, as well as cases with a previous diagnosis of cancer or simultaneous cancer diagnoses $(n=3200)$ were excluded from the analysis. Of the remaining cases, patients who underwent mastectomy $(n=296)$, did not undergo any surgery $(n=8)$, or cases where surgery information or pathologic tumour stage was incomplete or missing $(n=58)$ were not included in the present study. The final study cohort encompassed 1048 patients, who underwent breast conserving surgery for ductal carcinoma in situ over a 17-year period at two Breast Centres. Follow-up was completed on October 5th 2016.

\section{Statistical analyses and endpoints}

Statistical analyses were conducted using IBM SPSS Statistics 24.0 and $\mathrm{R}$ environment for statistical computing and visualization (version. 3.4.0). Patient and treatment characteristics were analysed using descriptive statistics and analysed using the Chi Square test. The percentages of the presented subcategories were related to the sum of available data of each variable and did not consider missing values. Primary endpoints were the impact of postoperative radiotherapy on local control and overall survival (OS). In-breast recurrence (IBR) was defined as invasive or in-situ recurrence in the ipsilateral breast. To account for competing risks, cumulative incidence analysis (CI) was used to calculate time to IBR and 
differences were assessed by Gray's Test for Equality of Cumulative Incidence Functions. OS was estimated using the Kaplan-Meier method and compared using the log-rank test. Relative survival was calculated by the ratio of the overall survival rate to the expected survival rate and $95 \%$ confidence intervals (95\%-CI) were used for assessing significance. To account for competing risks, Cox proportional hazards models were used to identify independent prognostic factors related to local recurrence free survival (LRFS) and OS. Furthermore, factors influencing the use of postoperative radiotherapy in DCIS were determined by using a multivariate logistic regression analysis. The significance level in all analyses was set at $5 \%$.

\section{Results}

Patient and treatment characteristics of 1048 patients diagnosed with ductal carcinoma in situ between 1998 and 2014 are summarized in Table 1. Median follow-up was 88.0 months; 69.0 months for the radiotherapy group and 123.0 months for patients receiving BCS alone. Overall, 388 patients underwent breast conserving surgery alone, while 660 patients were treated with additional postoperative radiotherapy of the ipsilateral breast. Age at diagnosis was significantly different between the groups $(p<0.001)$. Patients in the BCS group were significantly older, with a median age of 58.8 years (range: $30-90$ ), as compared to a median age of 57.3 years (range: 28-89) in patients undergoing BCS and postoperative radiotherapy. While tumour side was balanced between the two cohorts, there were a number of significant differences between patients undergoing $\mathrm{BCS}$ and patients receiving additional postoperative radiotherapy. High tumour grade (G3) was present in $29.3 \%$ of the surgery only group. The proportion was significantly higher in patients undergoing radiotherapy, presenting with $49.9 \%$ grade 3 tumours $(p<0.01)$. Similarly, negative hormone receptor status was more frequent in the radiotherapy group, as compared to patients not receiving radiotherapy $(17.3 \%$ vs $10.4 \%, p=$ 0.012 ). Nodal involvement (pN1) was present in 2 irradiated patients $(0.3 \%)$, while there was no case in the BCS group. Furthermore, patients in the radiotherapy cohort underwent surgical axillary intervention more frequently (sentinel lymph node biopsy or axillary dissection) as compared to the BCS cohort $(18.2 \%$ vs. $10.6 \%, p=$ 0.016). Regarding other treatment modalities, irradiated patients received endocrine therapy in $18 \%$. In contrast, in patients receiving no radiotherapy, adjuvant endocrine therapy was administered in $8.5 \%(p<0.001)$. A significant increase in the use of postoperative radiotherapy was documented over time (see Fig. 1). In 1998, only 42.9\% of patients received postoperative radiotherapy following $\mathrm{BCS}$, whereas the proportion increased to
Table 1 Cohort characteristics of the different treatment groups. BCS: breast conserving surgery

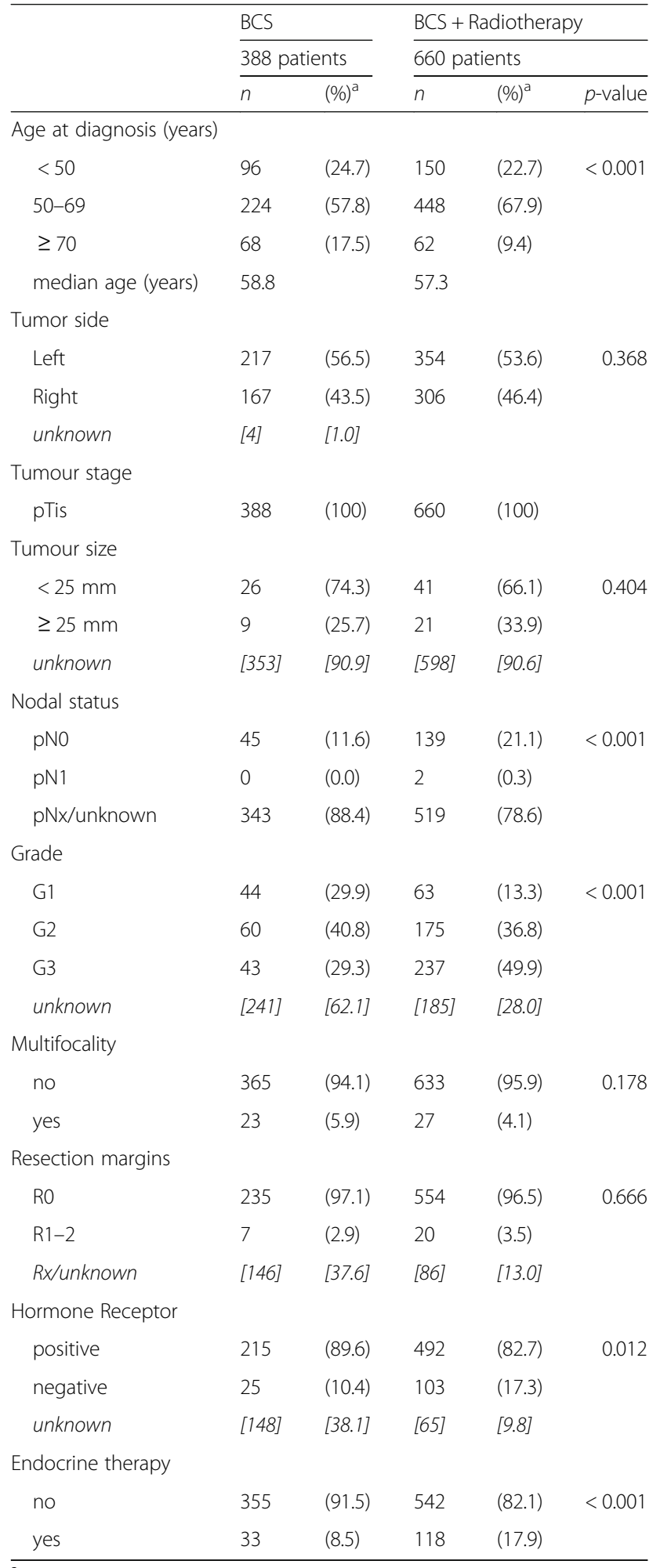

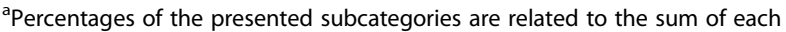
item with available data; missing values are not taken into account 


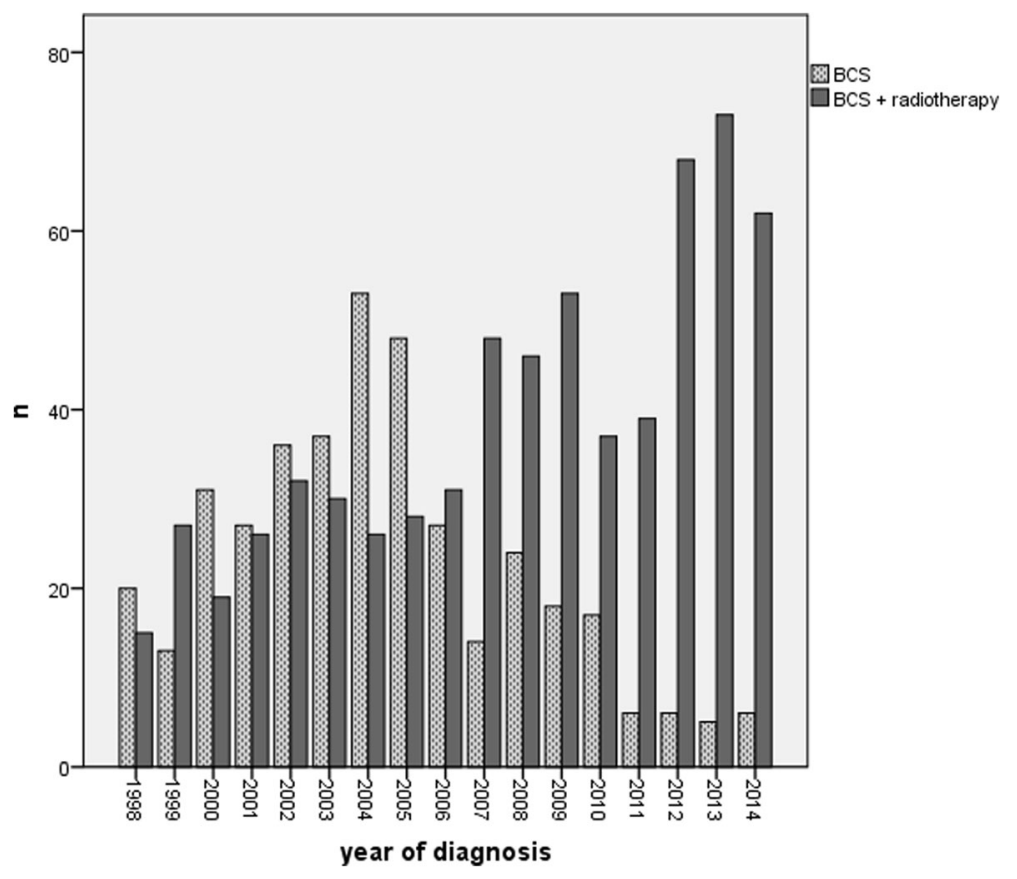

Fig. 1 The use of postoperative radiotherapy over time

$91.2 \%$ by 2014 . The standard radiotherapy regimen at the Department of Radiation Oncology of LMU University was whole-breast irradiation (50.4 Gy in 28 fractions or 50 Gy in 25 fractions) followed by a boost of 10-16 Gy to the tumour bed in high-risk cases with close resection margins.

During follow-up, 93 patients developed a DCIS recurrence and 50 patients an invasive ipsilateral recurrence. Median time to in-situ recurrence was 45 months (range: 5-149 months) and 69 months (range: 11-152 months) for invasive IBR. In-breast recurrence rates were significantly influenced by postoperative radiotherapy (Table 2). Cumulative incidence (CI) analysis showed a 10-year CI of IBR of $20.0 \%$ following BCS, compared to $13.6 \%$ in patients receiving postoperative radiotherapy $(p=0.012$, Fig. 2a). Overall, 10-year CI of DCIS IBR was $11.2 \%$ as compared to $6.5 \%$ for invasive recurrences. Other variables, including tumour age, size and grade, multifocality, resection status, hormone receptor status or the use of adjuvant endocrine therapy did not significantly influence outcome on univariate analysis. In multivariate Cox regression analysis accounting for confounding factors, postoperative radiotherapy remained independently associated with improved local recurrence free survival (hazard ratio [HR]: 0.579; 95\%CI: 0.384-0.872, $p=0.008$ ). Results are summarized in Table 3.

Patients receiving postoperative radiotherapy had a higher 10-year OS (96.7\%) compared to patients undergoing breast conserving surgery alone $(90.0 \%)$ ( $p=$ $0.001)$. In order to estimate disease-specific survival rates, relative survival was calculated. Ten-year relative survival was $105.4 \%$ for patients receiving postoperative radiotherapy and $101.6 \%$ without radiotherapy (Fig. 2b). After adjusting for risk factors in the Cox proportional hazards regression model, postoperative radiotherapy was not associated with improved overall survival (HR 0.526; 95\%CI: $0.263-1.052, p=0.069$ ). Other risk factors than age did not correlate with improved OS (Table 3).

\section{Factors associated with the use of postoperative radiotherapy}

A multivariate logistic regression analysis was performed to identify factors associated with the administration of postoperative radiotherapy in our collective (Table 4). Variables associated with the use of postoperative radiotherapy included young age at diagnosis, negative hormone receptor status, and administration of adjuvant endocrine therapy. Women aged $<50$ years were more likely to receive postoperative radiotherapy after BCS (OR 2.559, 95\%CI 1.416-4.625, $p<0.001)$. Similarly, women presenting with negative hormone receptor status had higher odds for being irradiated following BCS (OR 2. 625, 95\% CI 1. $458-4.728, p=0.001$ ). Furthermore, postoperative radiotherapy was more likely in patients receiving adjuvant endocrine therapy (OR 1. 762, 95\%CI 1.060-2.927, $p=$ 0.029).

\section{Discussion}

The present observational study analysed data from a large patient population treated at two major German breast centers outside of clinical trials. Women treated 
Table 2 Cumulative incidence of in-breast recurrences (IBR) and Kaplan-Meier estimates of overall survival (OS)

\begin{tabular}{|c|c|c|c|c|c|c|}
\hline & \multicolumn{3}{|l|}{ IBR } & \multicolumn{2}{|l|}{ OS } & \multirow[b]{2}{*}{$p$} \\
\hline & 5 y $(\%)$ & 10 y $(\%)$ & $p$ & 5 y $(\%)$ & 10 y $(\%)$ & \\
\hline \multicolumn{7}{|c|}{ Age at diagnosis (years) } \\
\hline$<50$ & 12.6 & 17.6 & 0.251 & 100.0 & 98.5 & $<0.001$ \\
\hline $50-69$ & 9.7 & 16.4 & & 98.2 & 95.1 & \\
\hline$\geq 70$ & 7.0 & 13.8 & & 88.1 & 74.6 & \\
\hline \multicolumn{7}{|c|}{ Tumour localisation } \\
\hline Left & 9.4 & 14.8 & 0.134 & 97.0 & 93.0 & 0.830 \\
\hline Right & 11.0 & 18.3 & & 97.6 & 93.8 & \\
\hline \multicolumn{7}{|l|}{ Tumour size } \\
\hline$<25 \mathrm{~mm}$ & 12.6 & 17.5 & 0.274 & 96.7 & 94.9 & 0.225 \\
\hline$\geq 25 \mathrm{~mm}$ & 6.7 & 6.7 & & 88.9 & 84.2 & \\
\hline \multicolumn{7}{|l|}{ Grade } \\
\hline G1 & 12.0 & 22.4 & 0.212 & 93.3 & 93.3 & 0.444 \\
\hline $\mathrm{G} 2$ & 5.3 & 12.4 & & 96.6 & 95.3 & \\
\hline G3 & 11.7 & 15.9 & & 99.4 & 90.7 & \\
\hline \multicolumn{7}{|l|}{ Multifocality } \\
\hline no & 10.0 & 16.2 & 0.592 & 97.3 & 93.4 & 0.972 \\
\hline yes & 11.1 & 19.4 & & 97.6 & 93.1 & \\
\hline \multicolumn{7}{|c|}{ Resection margins } \\
\hline RO & 9.3 & 15.6 & 0.526 & 97.6 & 93.1 & 0.953 \\
\hline R1-2 & 14.8 & 20.2 & & 96.2 & 96.2 & \\
\hline \multicolumn{7}{|c|}{ Hormone receptor status } \\
\hline positive & 8.6 & 16.2 & 0.719 & 97.2 & 94.3 & 0.501 \\
\hline negative & 13.0 & 15.6 & & 97.6 & 90.1 & \\
\hline \multicolumn{7}{|l|}{ Radiotherapy } \\
\hline no & 13.2 & 20.0 & 0.012 & 95.1 & 90.0 & 0.001 \\
\hline yes & 8.0 & 13.6 & & 99.1 & 96.7 & \\
\hline \multicolumn{7}{|c|}{ Endocrine therapy } \\
\hline no & 10.9 & 17.4 & 0.111 & 97.2 & 93.6 & 0.319 \\
\hline yes & 5.5 & 10.7 & & 97.6 & 92.3 & \\
\hline
\end{tabular}

with postoperative radiotherapy following BCS for DCIS of the breast were found to have a significantly lower rate of ipsilateral recurrences as compared to BCS alone (20.0\% vs $13.6 \%)$. This difference is consistent with other observational and randomised studies [3, 22, 23]. For example, the population-based SEER data analysis of 1103 women diagnosed with DCIS by Warren et al. [22], found a risk of developing an ipsilateral recurrence of $11 \%$ for patients who received postoperative radiotherapy versus $15 \%$ for women undergoing BCS alone, at a mean follow-up of 91 months. Accordingly, the NSABP B-17 trial [3] documented a 15-year cumulative incidence of ipsilateral recurrences of $19 \%$ for the lumpectomy group compared to $8 \%$ for the additional postoperative radiotherapy group. Similar results were seen in

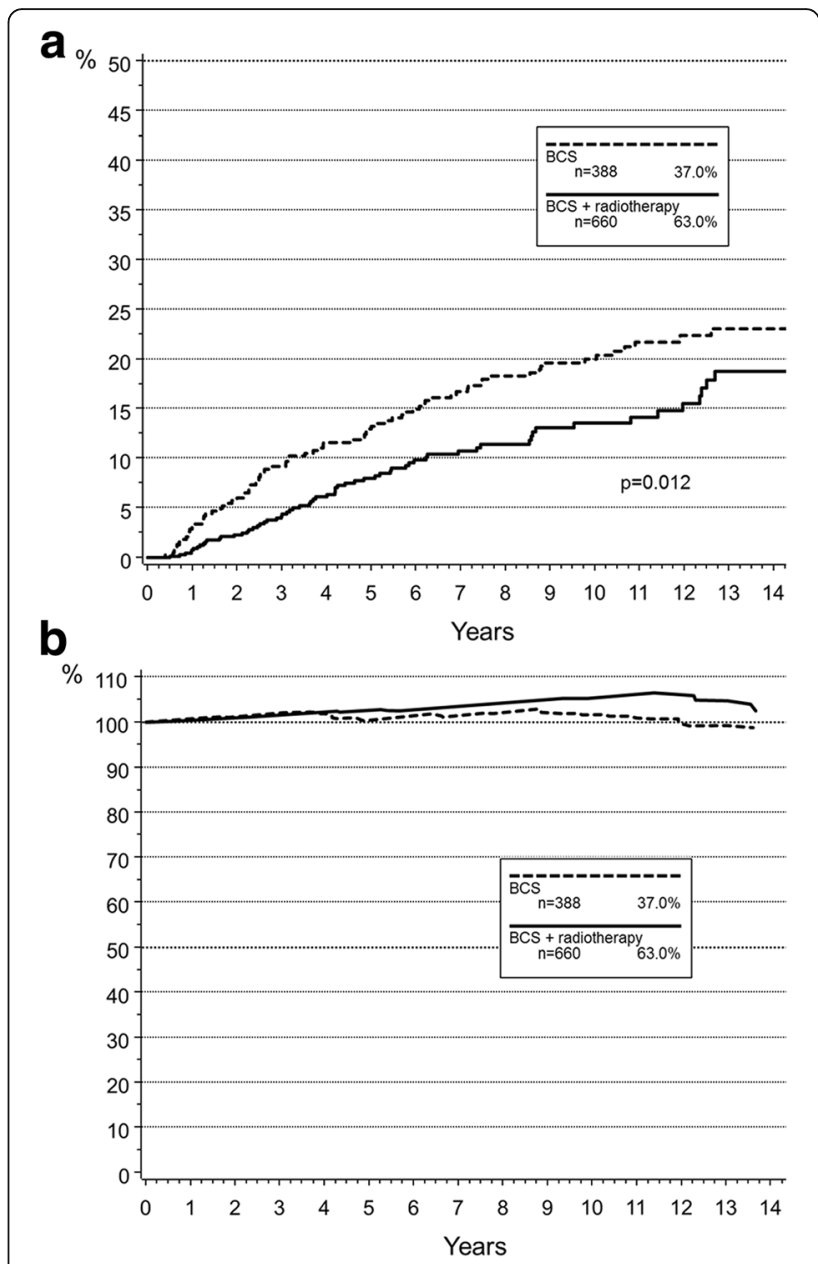

Fig. 2 Cumulative incidence of in-breast recurrences (a) and relative survival (b)

the EORTC 10853 trial [23], where radiotherapy reduced the incidence of invasive local recurrences after DCIS from $13 \%$ to $8 \%(p<0.01)$ after 10 years follow-up. The large EBCTCG meta-analysis [6] confirmed, that postoperative RT after breast conserving surgery approximately halves the risk of local recurrences as compared to surgical resection alone, with similar effects in reducing ipsilateral in situ and invasive recurrences.

It is well known, that postoperative RT reduces the risk of local recurrences for all subgroups, independent of age. Nevertheless, the EORTC trial [23] found that local recurrence rates were significantly influenced by age at diagnosis, and young age $<40$ years was identified as an independent high-risk factor for ipsilateral recurrences on multivariate analysis. Furthermore, the beneficial effect of RT on local control seems to be modified by age, resulting in a larger risk reduction for elderly patients. In the Swedish SweDCIS trial [2], women younger than 52 years had a smaller absolute risk reduction as compared to older age groups. The EBCTCG meta-analysis [6] confirmed that the proportional 
Table 3 Multivariate Cox regression analysis for loco-regional recurrence-free survival (LRFS) and overall survival (OS) for DCIS

\begin{tabular}{|c|c|c|c|c|c|c|}
\hline \multirow[b]{2}{*}{ Variable } & \multicolumn{3}{|l|}{ LRFS } & \multicolumn{3}{|l|}{ OS } \\
\hline & $\mathrm{HR}$ & $95 \% \mathrm{Cl}$ & p & $\mathrm{HR}$ & $95 \% \mathrm{Cl}$ & p \\
\hline Age at diagnosis & & & 0.089 & & & $<0.001$ \\
\hline$<50$ years & 1 & & & 1 & & \\
\hline $50-69$ y & 1.014 & $0.625-1.645$ & & 4.809 & $0.639-36.165$ & \\
\hline$\geq 70 y$ & 0.423 & $0.180-0.994$ & & 34.505 & $4.594-259.164$ & \\
\hline Radiotherapy & & & 0.008 & & & 0.069 \\
\hline yes & 0.579 & $0.384-0.872$ & & 0.526 & $0.263-1.052$ & \\
\hline no & 1 & & & 1 & & \\
\hline Multifocality & & & 0.818 & & & 0.790 \\
\hline yes & 1 & & & 1 & & \\
\hline no & 1.125 & $0.412-3.077$ & & 0.822 & $0.194-3.482$ & \\
\hline Resection margins & & & 0.424 & & & 0.948 \\
\hline RO & 1 & & & 1 & & \\
\hline R1-2 & 1.449 & $0.583-3.602$ & & 1.049 & $0.246-4.474$ & \\
\hline Endocrine Therapy & & & 0.097 & & & 0.274 \\
\hline yes & 1 & & & 1 & & \\
\hline no & 1.710 & $0.906-3.226$ & & 0.621 & $0.265-1.458$ & \\
\hline
\end{tabular}

Table 4 Adjusted Odd Ratios (OR) and 95\% Confidence Intervals (Cl) from the multiple logistic regression analysis for postoperative radiotherapy compared to omission of radiotherapy

\begin{tabular}{llll}
\hline Variable & OR & $95 \% \mathrm{Cl}$ & $p$ \\
\hline Age at diagnosis & & & $<0.001$ \\
$\quad<50$ years & 2.559 & $1.416-4.625$ & \\
$50-69$ y & 2.761 & $1.701-4.481$ & 0.403 \\
$\geq 70$ y & 1 & & \\
Tumour localisation & & & \\
$\quad$ Right & 1 & $0.597-1.230$ & \\
Left & 0.857 & & 0.778 \\
Multifocality & & $0.519-2.406$ & \\
no & 1.117 & & 0.001 \\
$\quad$ yes & 1 & & \\
Resection margins & & & \\
R0 & 1 & & \\
R1-2 & 1.290 & & \\
Hormone receptor status & & \\
Positive & 1 & & \\
Negative & 2.625 & & \\
Endocrine therapy & & $1.060-2.927$ & \\
no & 1.762 & & \\
yes & & & \\
\hline
\end{tabular}

reduction in the rate of ipsilateral breast recurrence achieved with radiotherapy was greater in older than in younger women. Lately, additional efforts have been undertaken to further increase local control rates for this "highrisk" group of young patients, for example by the addition of a boost to the tumour bed. A retrospective analysis of Moran et al. [10] showed that an additional RT boost had a significant benefit in decreasing in-breast recurrences in 4131 patients (HR 0.68, 95\%CI: $0.50-0.91, p=0.01$ ). Data from current randomised trials are eagerly awaited [24, 25]. It remains unclear, how many patients received a boost in the present study population, as detailed information on radiation dose was not available from cancer registry data.

The effect of radiotherapy on survival in ductal carcinoma in situ of the breast remains unclear. As DCIS is a non-invasive precursor lesion, overall survival after DCIS is very good, resulting in breast cancer specific survival rates of over $95 \%$ after 20 years [2]. So far, no statistically significant effect on breast cancer-specific survival has been observed in randomised trials [6]. It has to be mentioned, that most randomised trials were not powered to find a difference in breast-cancer specific survival or overall survival [13]. Furthermore, early detection and treatment of in situ recurrences (salvage mastectomy) might reduce the difference in outcome and result in comparable survival rates. On the other hand, it is well known, that patients with a subsequent invasive local recurrence have a significantly increased mortality risk after such events. In the EORTC trial [23], mortality risk increased by a factor of five and breast cancer-specific mortality by a factor of 17 following an invasive local 
recurrence. In contrast to randomised evidence, Sagara et al. [13] recently reported the outcome of a large population-based study of 32,144 DCIS patients. In this study, the use of radiotherapy in high-risk patients (high nuclear grade, young age, large tumor size) was associated with a significant survival benefit when compared to observation alone (HR 0.73, 95\% CI; $0.62-0.88, p=$ 0.003). After adjusting for risk factors in the Cox proportional hazards regression model in the present study, postoperative radiotherapy was not associated with improved overall survival (HR 0.526; 95\%CI: 0.263-1.052, $p=0.069)$. In order to estimate disease-specific survival rates, relative survival was calculated by adjusting the observed overall survival to the expected survival rates from the general population in Germany. Each individual of the study population was matched by age, sex and calendar year with the expected mortality of national life tables. Estimates of 10-year relative survival were 105\% for patients receiving postoperative radiotherapy. A relative survival higher than $100 \%$ indicates that non-cancer life expectancy was more favorable among patients within this study as compared to the general population. This "healthy user effect" is a known source of sampling bias in observational studies of early stage breast or prostate cancer [26-28]. Patients diagnosed with DCIS through a screening examination, generally tend to be healthier and have a longer life expectancy than the general population as they are concerned for their health, seek preventive services and might also partake in other healthy behaviors as well as follow medical advice.

The results have to be interpreted with caution, as any retrospective approach has inherent limitations: As known from other observational studies [15, 29], outcome in clinical effectiveness research is influenced by a number of unmeasured confounding factors and associations between treatments, and outcomes can result from confounding and have no causal correlation [30,31]. We tried to control for all known confounders available in the registry, which may have influenced the clinical treatment decisionmaking process. Nonetheless, it is not possible to fully control for host-related factors including comorbidities, performance status, or clinician- and patient-related preferences. Therefore, an unavoidable limitation is the inherent potential for selection bias due to disease severity [32], considering that the severity of the disease (e.g. presence of high-risk factors) is a potential confounder influencing the indication for postoperative radiotherapy in DCIS. In fact, the postoperative radiotherapy group of the present study included significantly more patients presenting with high-risk features like high tumour grade or negative hormone receptor status. Efforts have been undertaken to account for these clinicopathological risk-score features in multivariate analysis. However, a proper risk-group stratification to categorize baseline risks for local recurrence was not possible, since molecular subtypes and histopathological parameters were not available for all patients from the database. A strength of our study might be, that - in contrast to the highly selected and homogeneous study populations of randomised trials - this observational study reflects a heterogeneous cohort of patients treated in realworld clinical practice.

In the present analysis, a significant increase over time (1998-2014) regarding the use of postoperative radiotherapy was observed. This change in the use of RT probably results from the publication of four randomised trials conducted in the early nineties $[2-4,23]$. While mastectomy was the treatment of choice until the 1980s, these trials seem to have influenced treatment decisions in real-world clinical scenarios. Our analysis strongly confirms the change of treatment patterns: while in 1998 only $42.9 \%$ of patients received postoperative radiotherapy following BCS, this percentage has risen to $91.2 \%$ in 2014 . Factors associated with the use of postoperative radiotherapy on multivariate logistic regression analysis were typical high-risk features, as young age at diagnosis or negative hormone receptor status. Similar results were reported by Subhedar et al. [33], who retrospectively analysed 2996 cases of DCIS undergoing BCS from 1978 to 2010 at a single institution. Despite a significant increase of RT use over time $(p<0.0001)$, recurrence rates have fallen over time, even in patients undergoing BCS alone. The authors postulate that advances in digital mammography and improvements in pathological assessment might contribute to the reduction in recurrence rates [33].

Unfortunately, there are no widely accepted guidelines helping to stratify patients in clinical practice. Further prospective studies will be needed to clarify the debated role of RT in DCIS and assist clinicians to tailor risk-adapted RT strategies for their DCIS patients. In clinical practice, thorough counselling on the risk-benefit profile using prognostic scores $[12,13]$ or even multigene expression assays [34, 35] should be recommended for informed decision-making. The main challenge remains the selection of low-risk patients, in whom RT provides negligible absolute benefit and can be safely omitted, as well as to identify women at high risk, in order to be able to discuss advantages and disadvantages of a RT boost on an individual basis. Furthermore, patient compliance with follow-up care and the need for regular mammography surveillance play an important role in clinical decision making outside of randomised trials.

\section{Conclusion}

In conclusion, the present study provides insights regarding the adoption of postoperative RT following BCS for DCIS in a large cohort, reflecting "real-life" clinical practice in this setting. Postoperative RT was found to be associated with a reduced risk of ipsilateral recurrence and no survival benefit compared to observation alone. 


\section{Abbreviations}

BCS: Breast-conserving therapy; Cl: Confidence interval; DCIS: Ductal carcinoma in situ; IBR: In-breast recurrence; OR : Odds ratio; OS: Overall survival; RT: Radiotherapy

\section{Acknowledgements}

Not applicable.

\section{Availability of data and supporting materials \\ Not applicable.}

\section{Funding}

None.

\section{Authors' contributions}

SC and CB: conception and design of the study. SC, DR and SS performed statistical analysis and interpretation of data, and wrote the manuscript. SS and JE provided statistical support and data interpretation assistance. MP, SS, $D R, M N, U G, J E$ and $C B$ assisted with critically interpretation of data and revising the manuscript critically. MB, MP, SM and $\mathrm{NH}$ reviewed the manuscript with respect to gynaecological aspects. All authors read and approved the final manuscript.

\section{Ethics approval and consent to participate}

This retrospective study was exempt from requiring ethics approval. Bavarian state law (Bayrisches Krankenhausgesetz/Bavarian Hospital Law §27 Absatz 4 Datenschutz (Dataprotection)) allows the use of patient data for research, provided that any person's related data are kept anonymous. German radiation protection laws request a regular analysis of outcomes in the sense of quality control and assurance, thus in the case of purely retrospective studies no additional ethical approval is needed under German law.

\section{Consent for publication}

Not applicable.

\section{Competing interests}

The authors declare that they have no competing interests.

\section{Publisher's Note}

Springer Nature remains neutral with regard to jurisdictional claims in published maps and institutional affiliations.

\section{Author details}

'Department of Radiation Oncology, University Hospital, LMU Munich, Marchioninistraße 15, 81377 Munich, Germany. ${ }^{2}$ Department of Therapeutic Radiology and Oncology, Innsbruck Medical University, Innsbruck, Austria. ${ }^{3}$ Munich Cancer Registry (MCR) of the Munich Tumour Centre (TZM) at the Institute of Medical Information Processing, Biometry and Epidemiology (IBE), University Hospital, LMU Munich, Marchioninistr. 15, 81377 Munich, Germany. ${ }^{4}$ Red Cross Breast Centre, Taxisstr. 3, 80637 Munich, Germany. ${ }^{5}$ Department of Gynecology and Obstetrics, Breast Centre, University Hospital, LMU Munich, Marchioninistr. 15, 81377 Munich, Germany. ${ }^{6}$ Comprehensive Cancer Center (CCC-LMU), University Hospital, LMU Munich, Marchioninistr. 15, 81377 Munich, Germany.

Received: 5 October 2017 Accepted: 29 January 2018 Published online: 09 February 2018

\section{References}

1. Cutuli, Bruno, Jacques Bernier, and Philip Poortmans. 2014. Radiotherapy in DCIS, an underestimated benefit? Radiotherapy and Oncology. Elsevier Ireland Ltd. doi:https://doi.org/10.1016/j.radonc.2014.06.011.

2. Wärnberg F, Garmo H, Emdin S, Hedberg V, Adwall L, Sandelin K, Ringberg A, et al. Effect of radiotherapy after breast-conserving surgery for ductal carcinoma in situ: 20 years follow-up in the randomized SweDCIS trial. J Clin Oncol. 2014;32:3613-8. https://doi.org/10.1200/JCO.2014.56.2595.

3. Wapnir IL, Dignam JJ, Fisher B, Mamounas EP, Anderson SJ, Julian TB, Land $\mathrm{SR}$, et al. Long-term outcomes of invasive ipsilateral breast tumor recurrences after lumpectomy in NSABP B-17 and B-24 randomized clinical trials for DCIS. J Natl Cancer Inst. 2011;103:478-88. https://doi.org/10.1093/ jnci/djrO27.
4. Bijker, Nina, Philip Meijnen, Johannes L Peterse, Jan Bogaerts, Irène Van Hoorebeeck, Jean-pierre Julien, Massimiliano Gennaro, et al. 2006. Breast-conserving treatment with or without radiotherapy in ductal carcinoma-in-situ: ten-year results of European Organisation for Research and Treatment of Cancer randomized phase III trial 10853-a study by the EORTC breast cancer cooperative group and. J Clin Oncol 24: 3381-3387. doi:https:// doi.org/10.1200/JCO.2006.06.1366.

5. Houghton J. Radiotherapy and tamoxifen in women with completely excised ductal carcinoma in situ of the breast in the UK, Australia, and New Zealand: randomised controlled trial. Lancet. 2003;362:95-102. https://doi. org/10.1016/S0140-6736(03)13859-7.

6. Davidson N, Gelber R, Piccart M, Pruneri G, Pritchard K, Ravdin P, Robertson $J$, et al. Overview of the randomized trials of radiotherapy in ductal carcinoma in situ of the breast. Journal of the National Cancer Institute - Monographs. 2010;41:162-77. https://doi.org/10.1093/ jncimonographs/lgq039.

7. Smith GL. Toward minimizing overtreatment and undertreatment of ductal carcinoma in situ in the United States. J Clin Oncol. 2016;34:1172-4. https:// doi.org/10.1200/JCO.2015.66.2064.

8. McCormick B, Winter K, Hudis C, Kuerer HM, Rakovitch E, Smith BL, Sneige N, et al. RTOG 9804: a prospective randomized trial for good-risk ductal carcinoma in situ comparing radiotherapy with observation. J Clin Oncol. 2015;33:709-15. https://doi.org/10.1200/JCO.2014.57.9029.

9. Solin LJ, Gray R, Hughes LL, Wood WC, Lowen MA, Badve SS, Baehner FL, et al. Surgical excision without radiation for ductal carcinoma in situ of the breast: 12-year results from the ECOG-ACRIN E5194 study. J Clin Oncol. 2015;33:3938-44. https://doi.org/10.1200/JCO.2015.60.8588.

10. Moran, Meena S, Yinjun Zhao, Shuangge Ma, and et al. 2017. Association of radiotherapy boost for ductal carcinoma in situ with local control after whole-breast radiotherapy. JAMA Oncology.

11. Nilsson C, Valachis A. The role of boost and hypofractionation as adjuvant radiotherapy in patients with DCIS: a meta-analysis of observational studies. Radiother Oncol. 2015;114(Ireland):50-5. https://doi.org/10.1016/j.radonc. 2015.01.001.

12. Silverstein MJ. The University of Southern California/van Nuys prognostic index for ductal carcinoma in situ of the breast. Am J Surg. 2003;186:33743. https://doi.org/10.1016/S0002-9610(03)00265-4.

13. Sagara Y, Freedman RA, Vaz-Luis I, Mallory MA, Wong SM, Aydogan F, DeSantis S, Barry WT, Golshan M. Patient prognostic score and associations with survival improvement offered by radiotherapy after breast-conserving surgery for ductal carcinoma in situ: a populationbased longitudinal cohort study. J Clin Oncol. 2016;34:1190-6. https:// doi.org/10.1200/JCO.2015.65.1869.

14. Gennaro, Massimiliano, Maria Carmen De Santis, Luigi Mariani, Salvatore Lo Vullo, Vera Cappelletti, Roberto Agresti, Umberto Cortinovis, et al. 2017. Ten-year results of applying an original scoring system for addressing adjuvant therapy use after breast-conserving surgery for ductal carcinoma in situ of the breast. Breast 35. Netherlands: 63-68. doi:https://doi.org/10. 1016/.j.breast.2017.06.010

15. Corradini S, Bauerfeind I, Belka C, Braun M, Combs SE, Eckel R, Harbeck N, et al. Trends in use and outcome of postoperative radiotherapy following mastectomy: a population-based study. Radiother Oncol. 2016; https://doi. org/10.1016/j.radonc.2016.08.018.

16. Schönecker S, Walter F, Freislederer P, Marisch C, Scheithauer H, Harbeck N, Corradini S, Belka C. Treatment planning and evaluation of gated radiotherapy in left-sided breast cancer patients using the CatalystTM/ SentinelTM system for deep inspiration breath-hold (DIBH). Radiat Oncol. 2016;11:143. https://doi.org/10.1186/s13014-016-0716-5.

17. Teguh DN, Raap RB, Struikmans H, Verhoef C, Koppert LB, Koole A, Huang Y, van Hulst RA. Hyperbaric oxygen therapy for late radiation-induced tissue toxicity: prospectively patient-reported outcome measures in breast cancer patients. Radiat Oncol. 2016;11:130. https://doi.org/10.1186/s13014-0160700-0.

18. MCR. Munich Cancer Registry, http://www.tumorregister-muenchen.de/.

19. Jensen, O.M., D.M. Parkin, R. MacLennan, C.S. Muir, and R.G. Skeet. 1991. Cancer Registration: Principles and Methods. IARC Scientific Publication No. 95.

20. Tyczynski, J.E., E. Démaret, and D.M. Parkin. 2003. Standards and Guidelines for Cancer Registration in Europe. IARC Technical Publication No. 40.

21. DKG_DGGG. 2017. S3-Leitlinie Früherkennung, Diagnostik, Therapie und Nachsorge des Mammakarzinoms. Zuckschwerdt Verlag. 
22. Warren JL, Weaver DL, Bocklage T, Key CR, Platz CE, Cronin KA, Ballard-Barbash R, Willey SC, Harlan LC. The frequency of ipsilateral second tumors after breast-conserving surgery for DCIS: a population-based analysis. Cancer. 2005;104:1840-8. https://doi.org/10.1002/cncr.21406.

23. Donker M, Litiere S, Werutsky G, Julien JP, Fentiman IS, Agresti R, Rouanet $P$, et al. Breast-conserving treatment with or without radiotherapy in ductal carcinoma in situ: 15-year recurrence rates and outcome after a recurrence, from the EORTC 10853 randomized phase III trial. J Clin Oncol. 2013;31: 4054-9. https://doi.org/10.1200/JCO.2013.49.5077.

24. NCT00907868. Breast-Conserving Surgery and Whole-Breast Radiation Therapy With or Without Additional Radiation Therapy to the Tumor in Treating Women With Ductal Carcinoma in Situ.

25. NCT0047e0236. Radiation Doses and Fractionation Schedules in Non-low Risk Ductal Carcinoma In Situ (DCIS) of the Breast (DCIS).

26. Stroup AM, Cho H, Scoppa SM, Weir HK, Mariotto AB. The impact of state-specific life tables on relative survival. Journal of the National Cancer Institute - Monographs. 2014;2014:218-27. https://doi.org/10.1093/ jncimonographs/lgu017.

27. Howlader N, Ries LAG, Mariotto AB, Reichman ME, Ruhl J, Cronin KA. Improved estimates of cancer-specific survival rates from population-based data. J Natl Cancer Inst. 2010;102:1584-98. https://doi.org/10.1093/jnci/ dja366.

28. Giordano SH, Kuo Y-F, Duan Z, Hortobagyi GN, Freeman J, Goodwin JS. Limits of observational data in determining outcomes from cancer therapy. Cancer. 2008;112:2456-66 United States. https://doi.org/10.1002/cncr.23452.

29. Corradini S, Niyazi M, Niemoeller OM, Li M, Roeder F, Eckel R, Schubert-Fritschle G, et al. Adjuvant radiotherapy after breast conserving surgery - a comparative effectiveness research study. Radiother Oncol. 2014; 114:28-34. https://doi.org/10.1016/j.radonc.2014.08.027.

30. McGale P, Cutter D, Darby SC, Henson KE, Jagsi R, Taylor CW, Cutter D, McGale P, Taylor CW. Can observational data replace randomized trials? J Clin Oncol. 2016;34. United States::3355-7. https://doi.org/10.1200/JCO.2016.68.8879.

31. Giordano SH, Kuo Y-F, Duan Z, Hortobagyi GN, Freeman J, Goodwin JS, Foley NH, et al. Limits of observational data in determining outcomes from cancer therapy. Cancer. 2008;112:2456-66. United States. https://doi.org/10. 1002/cncr.23452.

32. Salas M, Hofman A, Stricker BH. Confounding by indication: an example of variation in the use of epidemiologic terminology. Am J Epidemiol. 1999; 149:981-3. https://doi.org/10.1093/oxfordjournals.aje.a009758.

33. Subhedar P, Olcese C, Patil S, Morrow M, Van Zee KJ. Decreasing recurrence rates for ductal carcinoma in situ: analysis of 2996 women treated with breast-conserving surgery over 30 years. Ann Surg Oncol. 2015;22:3273-81. https://doi.org/10.1016/j.meegid.2015.03.001.Transmissibility.

34. Solin LJ, Gray R, Baehner FL, Butler SM, Hughes LL, Yoshizawa C, Cherbavaz $\mathrm{DB}$, et al. A multigene expression assay to predict local recurrence risk for ductal carcinoma in situ of the breast. J Natl Cancer Inst. 2013;105:701-10. https://doi.org/10.1093/jnci/djt067.

35. Rakovitch E, Nofech-Mozes S, Hanna W, Baehner FL, Saskin R, Butler SM, Tuck A, et al. A population-based validation study of the DCIS score predicting recurrence risk in individuals treated by breast-conserving surgery alone. Breast Cancer Res Treat. 2015;152:389-98. Springer US. https://doi.org/10.1007/s10549-015-3464-6.

\section{Submit your next manuscript to BioMed Central and we will help you at every step:}

- We accept pre-submission inquiries

- Our selector tool helps you to find the most relevant journal

- We provide round the clock customer support

- Convenient online submission

- Thorough peer review

- Inclusion in PubMed and all major indexing services

- Maximum visibility for your research

Submit your manuscript at www.biomedcentral.com/submit

) Biomed Central 